\title{
Abstracts of Award-Winning Posters, 16th Annual Health Sciences Poster Conference, Faculty of Medicine, Health Sciences Centre, Kuwait University, Kuwait, May 3-5, 2011
}

\section{Dr. Nael Al-Naqeeb Undergraduate Research Award}

\section{Primary Caregiver and Child's Perception, Attitude and Coping in Paediatric Cancer \\ A.W. Al-Mogahwi, N.E. Bin Essa, D.M. Al-Sayed Ahmed, \\ S.O. Al-Zanki, R. Al-Lahu, M. Al-Shaleh, R.N. AlSabah \\ Faculty of Medicine, Kuwait University, Kuwait}

Introduction: Cancer is a disease that affects the body as well as the life of patients and families. The word 'cancer', especially in children, strikes fear in the minds of many people in Kuwait, which makes them avoid open and honest discussions on this topic. Our study attempted to shed some light on the quality of life, perception, and attitude of primary caregivers and children with cancer in $\mathrm{Ku}$ wait. Methods: This cross-sectional study enrolled paediatric cancer patients ages 4-18 years and their primary caregivers from three specialized cancer centres in Kuwait. Children were interviewed using the PedsQL, while their caregivers completed a self-report questionnaire which included among other questions the PedsQL-parent version and the Beck Depression Inventory-II. Results: Upon knowing their child's diagnosis most caregivers were either upset or afraid. The majority of children were not informed about their diagnosis and the most common reasons were that the child was young or it was better for the child not to know. The older the child, the more he or she was likely to be informed $(\mathrm{p}<0.001)$. Although most of the support received was from family members, the majority of caregivers needed support from medical staff. Female caregivers reported a decrease in their social duties and ability for self-care and reported more depressive symptoms. A slight agreement was found between child and caregiver evaluations of the PedsQL. Conclusions: Child and caregiver quality of life was greatly affected after the diagnosis of cancer. Many children were not informed of their cancer diagnosis or treatment plan. More support was needed from the medical staff.

Funding agency: None.

\section{Best Postgraduate Awards}

1. Basic Sciences (MSc)

\begin{abstract}
Autosomal Dominant Congenital Cataract of a Consanguineous Kuwaiti Family Revealed Genetic and Clinical Heterogeneity Discovered to Have Novel Mutations in CRYGB Gene

S. AlFadhlia , A. Sidky ${ }^{\mathrm{b}}$, A. Al-Hajri ${ }^{\mathrm{a}}$, A. Bahbahanib, c, F. Alkuraya ${ }^{\mathrm{d}}$

a Faculty of Allied Health Sciences, MLS, Kuwait University, ${ }^{b} \mathrm{Al}-$ Bahar Eye Center, Ministry of Health, and 'Department of Surgery, Faculty of Medicine, Kuwait University, Kuwait; dDepartment of Genetics, College of Medicine - Alfaisal University, King Faisal Specialist Hospital and Research Center, Riyadh, Saudi Arabia
\end{abstract}

Introduction: Congenital cataract is a clinically-genetically heterogeneous lens disorder. Members of a Kuwaiti family diagnosed with autosomal dominant congenital cataract (ADCC) across three generations were clinically characterized by complete eye examination and screened for causative mutation in CRYGB gene. Three phenotypes were identified: lamellar, anterior polar and complete cataract. Methods: DNA was extracted for all family members and subjected to whole genome linkage analysis (WGLA) using Affymetrix 250K Chip. WGLA of both healthy subjects and patients was compared. The locus with the highest LOD score was thoroughly screened for a potential gene related to lens development. The candidate gene was amplified using PCR, cloned, and sequenced to screen for the causative mutation. Results: WGLA showed that locus 2q34-36.1 harbors the CRYBA2 gene, with the highest LOD score of 1.5. Sequencing the CRYBA2 gene showed no alteration in the coding region or the exon-intron boundaries in patients. Therefore, locus 2q33-q37 with LOD score (1.49), harboring $\gamma$-crystallin gene clusters

\section{KARGER}

Fax +4161306 1234 E-Mail karger@karger.ch www.karger.com (c) 2011 S. Karger AG, Basel

1011-7571/11/0206-0585\$38.00/0

Accessible online at: www.karger.com/mpp 
CRYGA, B, C and D, was considered. All $\gamma$-crystallin genes were screened for mutation. Two novel heterozygous deletions were detected in CRYGB: intron 1 (58delG) and exon 2 (72delC) and a novel trinucleotide polymorphism in intron1 (11-13GGT $>$ AAA). Heterozygous intron 1 (58delG) was detected in 4 affected members with lamellar cataract. Heterozygous 72 delC was detected in 2 affected members, with either anterior polar or extreme cataract phenotype. One patient had a complete cataract found to be a compound heterozygote with $72 \mathrm{delC}$ and intron 1 (58delG) mutations. The novel, trinucleotide polymorphism in intron 1 (11-13GGT>AAA) was found to be maternally inherited to 2 affected daughters while the mother showed no clear eye abnormality. Therefore, the involvement of this polymorphism in ADCC in not clear. The $72 \mathrm{delC}$ mutation results in a truncated protein of 43 aa which preserved the first 24 aa of wild-type protein, a frame shift generating 19 random amino acids. Conclusions: We demonstrate the first complex mutation in the CRYGB gene in ADCC. The genetic heterogeneity resulted in clear clinical presentation of the disease. This case will advance our understanding of the genotypic-phenotypic correlation of ADCC.

Funding agency: Kuwait University; grants: YM10/09, GM01/01 and GM 01/05.

\section{Postgraduate Student Award (PhD)}

\section{Role of Human Metapneumovirus in Respiratory Tract Infections in Kuwait}

M. Al-Turab a , W. Chehadeh ${ }^{\mathrm{a}}$, F. Al-Mulla ${ }^{\mathrm{b}}$, W. Al-Nakib ${ }^{\mathrm{a}}$

Departments of a Microbiology and ${ }^{\mathrm{b}}$ Pathology, Faculty of Medicine, Kuwait University, Kuwait

Introduction: Human metapneumovirus (hMPV) has been recognized as an important cause of respiratory tract infections (RTIs) in all age groups and in all geographical areas. The role of hMPV in causing RTIs in Kuwait has not yet been investigated. The aim of the current study was to determine the prevalence of hMPV infection in Kuwait among patients with RTIs with respect to other respiratory viruses, and to identify the hMPV genotypes. Methods: During January to December 2009, 460 respiratory samples from 388 patients with RTIs were collected from different hospitals. They were tested for hMPV RNA by real-time PCR, and other respiratory viruses by conventional PCR. The type of detected hMPV RNA was identified by direct sequencing. Samples positive for H1N1 have been excluded from this study. Results: Out of 388 patients, 110 (28\%) were positive for viral respiratory infections; 21 (5.4\%) of them were positive for hMPV, 29 (7.5\%) were positive for rhinovirus, 13 (4\%) were positive for respiratory syncytial virus, and 10 (3\%) were positive for adenovirus. Most ( $n=19,90.5 \%$ ) of hMPV-positive patients were admitted to the ICU, $76 \%$ of them were of age 2 years and below, and $24 \%$ of age 59 and above. All hMPV-positive elderly patients have been diagnosed as having pneumonia while $50 \%$ of hMPV-positive infants had bronchopneumonia. Children with hMPV and rhinovirus coinfection $(n=3,1 \%)$ had recurrent chest infection and frequent
ICU admission. Genotype B2 was more prevalent than genotype $\mathrm{A}$, and the hMPV infection was mostly detected between December and May. Conclusions: This is the first study demonstrating the prevalence of hMPV infection in Kuwait (5.4\%) and it suggests that hMPV infection is prevalent in winter and spring months, and usually targets young children and aged individuals with lower RTI. The hMPV B2 is the predominate genotype in Kuwait.

Funding agency: Supported by College of Graduate Studies and Research Administration, Grant No. YM 05/09, Kuwait University.

\section{Postgraduate Student Award (Resident)}

\section{Patients Presenting to Secondary Emergency Services: A Comparison Study between Kuwait and Ireland}

M.A. Alali

Department of Medicine, University College Cork, Cork, Ireland

Introduction: Many patients attending accident and emergency (AE) departments in secondary care hospitals could be managed appropriately by general practitioners (GP). Inappropriate attendance of nonurgent patients to AE results in reduced efficiency of AE departments. This study attempts to compare patients attending two hospitals in two different countries. Methods: A questionnaire aided by interview was conducted between August 2006 and July 2007 including a total of 500 patients presenting to AE departments of Al-Amiri (Kuwait) and Cork University Hospital (CUH) in Ireland after excluding patients younger than 15 years. Sampling was by convenience and the data were analyzed using Statistical Package for the Social Sciences. Results: The majority of patients were between 25 and 64 years old. $63.6 \%$ of patients presenting to Al-Amiri Hospital were non-GP-referred compared to $58 \%$ of patients presenting to $\mathrm{CUH}(\mathrm{p}=0.2) .3 .6$ and $10 \%$ of patients came by ambulance in Kuwait and Ireland, respectively ( $p=0.004) .39 .6 \%$ of patients presenting to CUH had both hematological and radiological investigations while $10 \%$ had no investigations compared to 24 and $23.2 \%$ in Kuwait. In Kuwait, $48.8 \%$ presenting to AE did not require follow-up compared to $19.6 \%$ in Ireland ( $\mathrm{p}<0.001$ ), and only $14.8 \%$ were admitted in $\mathrm{Ku}-$ wait compared to $30.8 \%$ in Ireland. There was also a significant difference in patients detained for observation in the two groups (Kuwait: 1.6\%, Ireland: $17.2 \%, \mathrm{p}<0.001)$. Conclusions: Regardless of GP referral rate, data showed that the majority of Irish patients needed further follow-up, observation and admission, maybe suggesting more appropriate self- and GP-referral. In conclusion, primary health services need to be audited to assure the important role of GPs as gatekeepers of the health system in Kuwait.

Funding agency: None. 


\section{Basic and Applied Sciences Awards}

$$
1
$$

\section{Potentially Useful Interactions between Theophylline and Salbutamol on Cytokine Release in Human Monocytes}

\section{C.I. Ezeamuzie, P.K. Shihab}

Faculty of Medicine, Kuwait University, Kuwait

Introduction: $\beta_{2}$-Adrenoceptor agonists and theophylline are widely used bronchodilators. Unlike $\beta_{2}$ agonists, theophylline has additional anti-inflammatory effects. While $\beta_{2}$ agonists are commonly combined with inhaled steroids in the treatment of asthma, the effect of combining theophylline with $\beta_{2}$ agonists has not been studied. This study investigated the effect of theophylline and salbutamol, as well as their combination, on the release of tumor necrosis factor- $\alpha$ (TNF- $\alpha$ ) and interleukin- 6 (IL-6) from stimulated monocytes, and to compare this effect with that of dexamethasone and its combination with salbutamol. Methods: Highly purified human blood monocytes were pretreated with the drugs (alone or in combination) for $30 \mathrm{~min}$ before being stimulated in culture with $250 \mathrm{ng} / \mathrm{ml}$ lipopolysaccharide for $24 \mathrm{~h}$. Released TNF- $\alpha$ and IL- 6 were determined by ELISA. The corresponding mRNA expression for the two cytokines were also determined by RT-PCR using appropriate primers. Results: Salbutamol and procaterol $(\geq 0.1 \mu \mathrm{M})$ significantly inhibited the release of TNF- $\alpha$, but also enhanced that of IL- 6 . In contrast, theophylline $(30-100 \mu \mathrm{M})$ strongly inhibited the release of both cytokines. Interestingly, the combination of theophylline and salbutamol was additive in inhibiting TNF- $\alpha$ release but theophylline blocked the IL-6-enhancing effect of salbutamol. A similar effect was seen when dexamethasone was combined with salbutamol. These effects were also reflected in the mRNA expression of the cytokines, suggesting that the effects were genomic. Conclusions: $\beta_{2}$ agonists have opposing effects on the generation of TNF- $\alpha$ and IL-6, but when combined with theophylline, the latter, like dexamethasone, was capable of augmenting the anti-inflammatory effects of the $\beta_{2}$ agonists while preventing their proinflammatory effect. Thus, theophylline may have a potentially useful steroid-sparing effect.

Funding agency: Grant No. 2005-130-203, Kuwait Foundation for the Advancement of Sciences (KFAS).

\section{2 \\ Green Tea Extract (-)-Epigallocatechin-3-Gallate Promotes Spinal Neuroprotection and Axon Regeneration in Sciatic Nerve Crush Model of Nerve Injury: A Light and Electron Microscopic Study \\ W.M. Renno, M.S. Rao \\ Department of Anatomy, Faculty of Medicine, Kuwait University, Kuwait}

Introduction: (-)-Epigallocatechin gallate (EGCG) has been reported to have a neuroprotective and neuroregenerative role in the nervous system. Previously, we have shown functional and neurobehavioral improvements after chronic constrictive and sciatic nerve crush injuries. This study was designed to examine whether EGCG could improve survival of spinal neurons and restore the subcellular morphology of the sciatic nerve in a sciatic nerve crush model. Methods: Adult male Wistar rats $(n=8)$ were randomly assigned to: (i) sham control (SC), (ii) sciatic nerve crush + saline $(\mathrm{SNC}+\mathrm{S})$ and (iii) sciatic nerve crush + EGCG $(\mathrm{SNC}+\mathrm{EGCG})$ groups. In SNC + S and SNC + EGCG groups, the sciatic nerve was exposed and crushed with forceps for $60 \mathrm{~s}$ and treated with saline (i.p.) or EGCG (50 mg/kg), respectively, for 2 weeks. Lumbosacral spinal cord (LSC) and nerve segments distal to the crush were harvested, processed for light and electron microscopy (EM). Results: The ventral gray horn of LSC showed irregular, darkly stained neurons and substantial reduction in the number of neurons ipsilateral to the crush injury in the SNC $+\mathrm{S}$ group. Such degenerative changes were not seen in the SNC + EGCG group. The contralateral side of the SNC + EGCG group was comparable to the contralateral side of $\mathrm{SNC}+\mathrm{S}$ and $\mathrm{SC}$ groups. Morphometric analysis of the sciatic nerve in the SNC + $S$ group showed significant increase in the number of myelinated fibers compared to the SC group. EGCG treatment significantly decreased the number of myelinated axons compared to SNC $+\mathrm{S}$. EM revealed normal appearance of regenerated fibers, normal thickness of myelin sheaths and axons in the SNC + EGCG group. The axons in the EGCG-treated group also had normal SchmidtLantermann clefts. The extracellular matrix was comparable to normal collagen fibers with well-organized distribution and absence of disintegrated myelin figures. Conclusions: EGCG protects spinal neurons and enhances subcellular recovery subsequent to peripheral nerve injury and thus improves nerve regeneration.

Funding agency: Department of Anatomy, Faculty of Medicine, Kuwait University.

\section{Clinical Sciences Awards}

1

\section{Prospective Longitudinal Study of Factors Associated with Progression/Regression of Microalbuminuria in Patients with Type 2 Diabetes Mellitus}

O.A. Mojiminiyia , N.A. Abdellab , D. Al-Khrayefa, F. Al-Mulla ${ }^{\mathrm{a}}$, C. Pinto ${ }^{b}$, S. George ${ }^{\mathrm{a}}$, H. Al-Mohammedic

Departments of a Pathology and ${ }^{\mathrm{b}}$ Medicine, Faculty of Medicine, Kuwait University, and ' Department of Medicine, Mubarak Al-Kabeer Hospital, Kuwait

Introduction: Microalbuminuria (MA) is a predictor of the progression of diabetic nephropathy and an independent risk factor for coronary heart disease (CHD). Regression of MA is desirable in diabetic subjects but the factors associated with regression or progression of MA have not been studied in the Kuwaiti population. The aims of this study were to estimate the frequency and 
factors associated with progression of MA. Methods: 123 (72 F, 51 M) patients were followed up for a median of 5.5 years. During the follow-up period, MA was assessed 3 times a year on separate visits. We evaluated whether MA had progressed (mean $\geq 50 \%$ of baseline mean), had remained unchanged, or had regressed (mean $\leq 50 \%$ of baseline mean). Multiple regression analysis was used to determine the predictive values of sex, age, duration of diabetes, CHD, baseline cystatin C (CC), baseline high-sensitivity C-reactive protein (hs-CRP), leptin, adiponectin, mean $\mathrm{HbA}_{1 \mathrm{c}}$, diastolic blood pressure, systolic blood pressure and the insertion/deletion polymorphism of the Angiotensin-Converting Enzyme (ACE) gene for the progression of MA. Results: At baseline, $49.5 \%$ were normoalbuminuric and $50.5 \%$ were microalbuminuric; at the end of follow-up, $64 \%$ had no change; $10 \%$ regressed and $26 \%$ progressed. Mean hs-CRP, leptin, $\mathrm{HbA}_{1 \mathrm{c}}$ and $\mathrm{CC}$ were higher and mean adiponectin lower in patients with progression compared to those who regressed or had no change. Odds ratios (OR) for significant factors in progression are: CHD (1.2); CC (10.4); hsCRP (1.5); mean $\mathrm{HbA}_{1 \mathrm{c}}$ (1.2) and ACE I/D DD genotype (11.5). All MA patients with CC $>1.4 \mathrm{mg} / \mathrm{l}$ had progression of MA with OR of 26.1 , whereas patients with $C C<1.1 \mathrm{mg} / \mathrm{l}$ had regression with OR of 2.0. Conclusions: ACE I/D genotype and CC at baseline are the most significant of the multiple determinants of the progression/regression of MA. These findings have significant implications for clinical practice as prevention of MA is an important therapeutic target.

Funding agency: KU research admin grant MG 033.

\section{2 \\ Expression of Progesterone Receptor and Its Isoforms PRAB and PRB in Fine-Needle Aspirates from Breast Carcinoma}

K. Kapila, J.T. Anim, I.M. Francis, S.S. George, A. Behbehani, F. Al Mulla

Faculty of Medicine, Kuwait University, Kuwait

Introduction: Progesterone receptor (PR) is a surrogate member of a functional estrogen receptor (ER) and is mediated by two functionally different isoforms, PRAB and PRB. The relative levels of PRAB and PRB within target cells may determine the functional response of $\mathrm{PR}$. Little is known regarding the expression of PRAB and PRB in breast carcinomas. The aim of this study is to evaluate the expression of $\mathrm{PR}, \mathrm{PRAB}$ and $\mathrm{PRB}$ in fine-needle aspirates (FNA) from breast carcinoma and correlate it with its expression in tissue sections and other prognostic parameters such as cytologic grading, ER, C-erb B2 and Ki-67. Methods: In 40 cases of breast carcinoma with available material for cytohistologic correlation, PR, PRAB and PRB were studied by immunocytochemical methods. Their expression in aspirates and tissue sections were correlated with cytologic grading, ER, C-erb B2 and Ki-67. Results: PR and its isoforms PRAB and PRB were demonstrated by immunocytochemistry on FNA smears in 43, 69 and $7 \%$ of grade 1 carcinomas and 78,33 and $22 \%$ of grade 3 carcino- mas, respectively. In tissue sections PR, PRAB and PRB were demonstrable in 88,38 and $19 \%$ of grade 1 carcinomas in contrast to 40,0 and $20 \%$ of grade 3 carcinomas, respectively. No significant correlation was observed between the grade of the tumor and PR expression. PR-positive breast carcinoma cases were positive for PRAB (63\%) and PRB (19\%). This corroborated with tissue section findings. PR, PRAB and PRB were positive in 55, 45 and $15 \%$ of Er $\alpha$-negative cases, respectively. In tissue sections there was marginal PR (25\%) and PRAB (12\%) positivity in Er $\alpha$-negative tumors. No statistical correlation between Ki-67 and Cerb B-2 with ER and PR status of the tumor was observed in the cases studied. Conclusions: The expression of $\mathrm{PR}$ and its isoforms PRAB, PRB was concordant with their expression in tissue sections in the majority of the cases. No statistical correlation was found with C-erb B2 and Ki-67.

Funding agency: Kuwait University Research Grant MG01/04.

\section{3 \\ Features of Polycystic Ovarian Syndrome in Adolescent Women and Management with Metformin}

M.K. Al-Azemi, W. Al-Jassar, F.E. Omu, A.E. Omu

Department of Obstetrics and Gynecology, Faculty of Medicine and Maternity Hospital, and College of Nursing, PAAET, Kuwait

Introduction: Polycystic ovary syndrome (PCOS) is characterized by anovulation in the form of irregular or absent menstrual periods and hyperandrogenism manifesting as elevated androgens with hirsutism, acne and deepening voice. Adolescent PCOS presents a serious treatment challenge because the features are like those of pubertal changes. Objective: To investigate the clinical features of PCOS in adolescent women and the role of metformin and exercise in the management. Methods: Twentyseven adolescent women, between 10 and 20 years of age with anovulation and hyperandrogenism, were recruited from the outpatient clinic. All had clinical (history and physical examination) and biochemical (hormone and lipid profiles and sex hormonebinding globulin, SHBG) evaluation and were randomized into three treatment groups: metformin only, metformin and exercise and exercise only and followed up for 3 months. Results: The features of PCOS included oligomenorrhea, high BMI and acne like in other parts of the world. All three options resulted in weight reduction and regular menstrual periods in about $34 \%$, respectively. Metformin with exercise had a more significant effect in decreasing total testosterone (50\%) as compared to $28 \%$ with metformin only, $14 \%$ with exercise only. Similarly, there was an increase in high-density lipoprotein (44\% to 20 and $12 \%$ ) and SHBG, but no effect on hormone profile. Conclusions: Metformin and exercise individually resulted in significant weight reduction and regular periods, but combined metformin and exercise was better at reducing total testosterone and increasing high-density lipoprotein.

Funding agency: None. 


\section{Case Report Award}

\section{Signet Ring Cell Adenocarcinoma of the Rectum with Bilateral Breast Metastasis: A Case Report and Review of the Literature}

H. Shaaban a , M. El Kabany a , H.G. Amanguno , H.G. Hebbar ${ }^{\text {, }}$ A. Jassar', I.M. Francis', K. Kapilac

Departments of a Pathology and ${ }^{b}$ Radiology, Hussein Makki Al Juma Center for Specialized Surgery, and 'Department of Pathology, Faculty of Medicine, Kuwait University, Kuwait

Background: Breast metastases from non-breast primaries are rare, constituting about $2 \%$ of all breast metastases in female patients. Lymphoma, metastatic melanoma and bronchial carcinoma are the malignancies that account for the majority of cases. Breast metastases from colorectal carcinoma have been described in a small number of cases in the literature. Case Summary: A 30 -year-old pregnant woman presented to Al Amiri Hospital with a circumferential rectal mass in February 2010. Biopsy was reported as signet ring cell carcinoma (the origin of which was not known). The patient was referred to Kuwait Cancer Control Center for preoperative chemoradiotherapy. After four radiotherapy sessions, MRI was done which revealed multiple bone metastases with a locally advanced rectal mass. Carcinoembryonic antigen and cancer antigen 19-9 were elevated. Then she developed intestinal obstruction, which was relieved by a diverting colostomy. In November 2010 the patient presented with bilateral retroareolar breast lumps. Ultrasound revealed well-defined mass lesions. Cytology of both breast nodules showed loosely cohesive tumor cells with moderate pleomorphism, vesicular nuclei and intracytoplasmic vacuoles. Signet ring cells were also seen in a mucinous background. On morphology the possibility of a lobular carcinoma or metastasis from the rectal carcinoma was considered. Tumor cells in both breasts stained positive for ER and PR receptors, CK7, CDX2, CEA and E-cadherin and were focally positive for CK20. Cells stained negative for GCDFP-15. However, a final diagnosis of mucinous carcinoma, metastatic from primary colorectal carcinoma, was entertained. Conclusion: Although colorectal metastasis to breast is rare, it should be included in the differentials for any patient with a known history of colorectal cancer. Accurate differentiation is necessary because treatment differs significantly for patients with breast metastasis as compared with patients with a second primary breast cancer. 